\title{
Associação de condições socioeconômicas, saúde bucal, hábitos orais e má oclusão com 0 desempenho escolar de escolares de 5 anos
}

\author{
Association between socioeconomic conditions, oral health, oral habits, and \\ malocclusion with school performance in 5 -year-old students
}

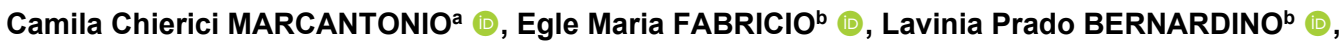 \\ Marília Narducci PESSOA ${ }^{b}$ (1), Eloisa MARCANTONIO ${ }^{\text {b* }}$ (1) \\ aUNESP - Universidade Estadual Paulista, Faculdade de Odontologia, Departamento de Diagnóstico e Cirurgia, \\ Araraquara, SP, Brasil \\ 'UNIARA - Universidade de Araraquara, Faculdade de Odontologia, Departamento de Ortodontia, Araraquara, SP, Brasil
}

Como citar: Marcantonio CC, Fabricio EM, Bernardino LP, Pessoa MN, Marcantonio E. Associação entre condições socioeconômicas, saúde bucal, hábitos orais e má oclusão com o desempenho escolar de escolares de 5 anos. Rev Odontol UNESP. 2021;50:e20210054. https://doi.org/10.1590/1807-2577.05421

\begin{abstract}
Resumo
Introdução: Problemas de origem dentária podem ocasionar um forte impacto em aspectos funcionais, emocionais e sociais das crianças, comprometendo-as em âmbito escolar, na aprendizagem e no seu desenvolvimento fisiológico. Objetivo: Analisar as associações entre as variáveis de condições socioeconômicas, saúde bucal, hábitos e má oclusão com o desempenho escolar das crianças de cinco anos. Material e método: A amostra consistiu em 753 crianças matriculadas em escolas públicas da cidade de Araraquara-SP. Foram realizados exames bucais para avaliar a presença de cárie dentária e má oclusão. Foram analisados, também, hábitos de sucção e bruxismo, condição socioeconômica e a qualidade de vida relacionada à saúde bucal. Os dados foram avaliados pelas análises descritivas e por modelos de regressão logística, simples e múltiplo $(p \leq 0,05)$. Foram estimados os Odds Ratios, com intervalos de $95 \%$ de confiança. Resultado: Apresentaram chances de ter pior desempenho escolar: crianças de famílias com menores rendas (1,76 vez), crianças que apresentaram piores condições de saúde bucal (1,93 vez), crianças que apresentaram um comportamento deficiente em relação à higiene oral (6,6 vezes) e crianças do sexo masculino (1,48 vez). Não houve evidências de associação entre as variáveis de saúde bucal, má oclusão e de hábitos orais deletérios com o desempenho escolar percebido pelos responsáveis. Conclusão: Crianças de famílias com menores rendas, que recebem ajuda financeira do governo, com pior avaliação da saúde bucal e higiene oral, do sexo masculino e que são cuidadas por pessoas que não os pais apresentaram maior chance de ter o desempenho escolar comprometido.
\end{abstract}

Descritores: Desempenho escolar; saúde bucal; má oclusão; hábitos; situação socioeconômica.

\begin{abstract}
Introduction: Problems of dental origin can cause a strong impact on functional, emotional and social aspects of children, compromising them at school, in learning and in their physiological development. Objective: Evaluate the associations between variables of socioeconomic conditions, oral health, habits and malocclusion with the school performance of 5-year-old children. Material and method: The sample consisted of 753 children enrolled in public schools in the city of Araraquara-SP. Oral examinations were performed to evaluate the presence of dental caries and malocclusion. We also analyzed sucking habits, bruxism, socioeconomic status and quality of life related to oral health. Data were evaluated by descriptive analyses and logistic regression models, simple and multiple ( $\leq \leq 0.05)$. Odds Ratios (OR) were estimated, with 95\% confidence intervals. Result: Children from families with lower income (1.76 times), children with worse oral health conditions (1.93 times), children with poor oral hygiene behavior (6.6 times), and male children (1.48 times) were more likely to have worse school performance. There was no evidence of association between the variables of oral health, malocclusion and deleterious oral habits with the school performance perceived by the guardians. Conclusion: Children from families with lower incomes, who receive
\end{abstract}


financial help from the government, with worse evaluation of oral health and oral hygiene, males and who are cared for by people other than their parents were more likely to have their school performance compromised.

Descriptors: Academic performance; oral health; malocclusion; habits; socioeconomic status.

\section{INTRODUÇÃO}

A infância é um período em que há aquisição de novos conhecimentos e hábitos, os quais refletem posteriormente nos comportamentos relacionados à saúde. 0 padrão de higiene em préescolares tem influência de diversos fatores, como: alto consumo de açúcar, pouco acesso à água e a instalações sanitárias seguras, dificuldade aos acessos de serviços de saúde bucal e escassez de cuidados primários de saúde com promoção da higiene bucal e técnicas adequadas ${ }^{1}$. Crianças e adolescentes que possuem alguma doença dentária podem estar em desvantagem quanto ao desenvolvimento fisiológico, fornecendo evidências de que a influência da condição de saúde bucal e a percepção negativa dos pais sobre a saúde das crianças comprometem o desempenho escolar** 0 desempenho escolar se relaciona diretamente com os fatores socioeconômicos, pois a baixa condição econômica interfere tanto na saúde bucal quanto na autopercepção e na qualidade de vida. É demonstrada uma forte associação entre problemas bucais e desempenho escolar, uma vez que a condição bucal interfere na autoestima, na socialização e na concentração escolar ${ }^{2}$.

Assim como a saúde bucal, alguns hábitos podem ter influência sobre a qualidade de vida das crianças. A permanência de um hábito por dois anos seguidos pode torná-lo deletério e as alterações que ele pode causar na estrutura e nas funções do sistema estomatognático dependem de sua intensidade, frequência e duração ${ }^{3}$. Dentre eles, os mais comuns são: bruxismo; respiração bucal; sucção digital, labial, de chupeta e de mamadeira; onicofagia, e hábito de morder o lábio. Esses hábitos resultam em alterações de tecidos musculares, dentários e ósseos, causando alteração no padrão normal de crescimento, comprometendo a oclusão e interferindo nas funções de fala, mastigação e respiração, sem contar no comprometimento orofacial, que pode gerar vários problemas de convivência social em crianças e adolescentes. ${ }^{3}$ Nessa perspectiva, se faz necessária uma conscientização dos pais e/ou responsáveis, bem como dos professores, para que programas e estratégias de retirada de hábitos sejam realizados o mais precoce possível, no intuito de contribuir para o desenvolvimento físico e emocional dos envolvidos ${ }^{4}$.

Em casos de hábitos que causam desvio do padrão respiratório, há uma influência negativa na estrutura dento-facial e esquelética, principalmente durante a fase de crescimento da criança. As principais consequências são: crescimento facial vertical, palato ogival, face estreita, maxilares pouco desenvolvidos, narinas estreitas e/ou inclinadas para cima, menor espaço na cavidade nasal, desvio de septo, classe II, overjet, mordida cruzada e/ou aberta, e protrusão de incisivos superiores. Além disso, a respiração bucal causa sonolência e cansaço, refletindo na concentração e no raciocínio, e tornando os envolvidos resistentes às tarefas físicas e mentais contínuas, trazendo comprometimento na aprendizagem e no humor ${ }^{5}$.

Dentro desse panorama, o que se observa é um comprometimento da saúde geral, podendo levar a uma deterioração da qualidade de vida. Nesse sentido, considerando as variáveis citadas acima, o intuito deste trabalho foi verificar de que forma as condições socioeconômicas, de saúde bucal, de hábitos orais e de má oclusão podem influenciar no desempenho escolar de crianças.

** Quadros LN. Condições de saúde bucal e sua relação com desempenho e absenteísmo escolar de crianças e adolescentes: uma revisão sistemática [dissertação]. Manaus: Universidade Federal do Amazonas; 2019. 


\section{MATERIAL E MÉTODO}

Este é um estudo observacional transversal que foi desenvolvido na cidade de Araraquara, São Paulo, Brasil com escolares de cinco anos de idade matriculados em Centros de Educação e Recreação (CERs) municipais. 0 estudo foi previamente aprovado pelo Comitê de Ética e Mérito Científico sob CCAE número 2.814.397. Foi realizada uma amostragem probabilística por conglomerados para se fazer a seleção das escolas. Erro amostral de 5\%, CPOD = 1,99 (dado para região Sudeste - Brasil, 2010), taxa de não resposta (perda de elementos amostrais) de $20 \%$ e nível de confiança de $95 \%$ foram utilizados para calcular o tamanho da amostra.

Como isso, totalizaram-se 38 escolas englobando 2.114 crianças e, então, após o cálculo de amostragem, foi obtido o total de 753 escolares de cinco anos, que participaram do estudo. Como critério de inclusão, os alunos deveriam possuir Termo de Consentimento Livre e Esclarecido (TCLE) assinado pelos pais ou responsáveis. Como critério de exclusão, os alunos não deveriam apresentar doenças sistêmicas, hipoplasia severa, fazer uso de aparelho ortodôntico e/ou tratamento ortodôntico prévio, e não deveriam apresentar problemas físicos ou psíquicos que pudessem impedir a comunicação e a realização dos exames.

As análises foram realizadas por examinadores calibrados por meio do coeficiente de KAPPA $(\mathrm{K}=0,91)^{6}$. Os exames clínicos foram realizados nas escolas, por examinadores previamente treinados. Entretanto, para as concordâncias intraexaminadores, $10 \%$ da amostra foi reexaminada durante a coleta de dados.

Foram analisados os seguintes desfechos: cárie dental (avaliada através de índices CPOD OMS, 19997); alterações oclusais (avaliação dos arcos dentários na fase de dentadura decídua e em relação sagital anteroposterior, relação transversal na região posterior, relação vertical na região anterior e relação horizontal anterior) ${ }^{8}$; presença de hábitos de sucção, distúrbio de respiração, alergias, trauma dental e tratamento ortodôntico (avaliados pelo questionário adaptado de Dimberg et al. ${ }^{9}$; impacto na qualidade de vida (avaliada por meio do Oral Health Impact Scale ECOHIS); condições socioeconômicas e demográficas [coletadas pelo questionário estruturado de Meneghim et al. ${ }^{10}$.

As análises das associações das variáveis independentes com o desempenho escolar foram realizadas por modelos de regressão logística (simples e múltiplo). As variáveis que apresentaram $\mathrm{p} \leq 0,20$ foram inseridas no modelo, mas compuseram o modelo final somente aquelas com $\mathrm{p} \leq 0,05$. Foi estimada a Odds Ratio (OR) com intervalo de confiança de $95 \%$ (ORIC95\%), verificada a significância do modelo $\left(G^{2}\right)$ e computado o valor do Pseudo- $R^{2}$ de Mcfadden. Os outliers foram investigados com base nos resíduos (z-residual). 0 ajuste do modelo foi avaliado pelo Critério de Informação de Akaike (AIC) e -2 Log L (log likelihood). As análises foram realizadas com auxílio do programa SAS (Statistical Analysis System, Cary, NC, EUA), adotando-se nível de significância de 5\%.

\section{RESULTADO}

Foi incluído no estudo um total de 753 escolares de cinco anos de idade pertencentes à rede municipal da cidade de Araraquara, São Paulo, Brasil, que se distribuíram em 51,9\% do sexo feminino e $48,1 \%$ do sexo masculino. Quanto às características sociodemográficas, pode-se observar que $82,5 \%$ das crianças pertenciam a famílias com renda de até cinco salários-mínimos, $73,3 \%$ a famílias com até quatro pessoas, $64,7 \%$ com pais com pelo menos o Ensino Médio completo e 69,3\% com mães com pelo menos o Ensino Médio completo. Além disso, 19,9\% eram de famílias que recebem algum tipo de auxílio financeiro do governo e 45,2\% residem com os pais, sendo ambos empregados. Além disso, a maioria dos responsáveis classificou o desempenho dos escolares nos últimos 12 meses em excelente ou muito bom $(66,1 \%)$ (Tabela 1). 
Tabela 1. Perfil sociodemográfico e desempenho escolar de escolares de cinco anos de Araraquara, São Paulo, Brasil, nos últimos 12 meses, pela percepção dos responsáveis $(n=753)$

\begin{tabular}{|c|c|c|c|}
\hline Variável & Categoria & Frequência & $\%$ \\
\hline \multirow[t]{2}{*}{ Sexo } & Feminino & 391 & 51,9 \\
\hline & Masculino & 362 & 48,1 \\
\hline \multirow[t]{7}{*}{ Renda da família } & Até $1 \mathrm{SM}$ & 148 & 19,6 \\
\hline & De 1 a 2 SM & 167 & 22,2 \\
\hline & De 2 a 3 SM & 156 & 20,7 \\
\hline & De 3 a 5 SM & 151 & 20,0 \\
\hline & De 5 a 7 SM & 71 & 9,4 \\
\hline & De 7 a 10 SM & 46 & 6,1 \\
\hline & Acima de 10 SM & 14 & 1,9 \\
\hline \multirow[t]{6}{*}{ Número de pessoas } & Até 2 & 31 & 4,1 \\
\hline & 3 & 235 & 31,2 \\
\hline & 4 & 286 & 38,0 \\
\hline & 5 & 127 & 16,9 \\
\hline & 6 & 41 & 5,4 \\
\hline & Acima de 6 & 33 & 4,4 \\
\hline \multirow[t]{10}{*}{ Grau de instrução do pai } & Não alfabetizado & 13 & 1,7 \\
\hline & Alfabetizado & 33 & 4,4 \\
\hline & Ensino Fundamental I incompleto & 21 & 2,8 \\
\hline & Ensino Fundamental I completo & 20 & 2,7 \\
\hline & Ensino Fundamental II incompleto & 35 & 4,6 \\
\hline & Ensino Fundamental II completo & 70 & 9,3 \\
\hline & Ensino Médio incompleto & 74 & 9,8 \\
\hline & Ensino Médio completo & 345 & 45,8 \\
\hline & Superior incompleto & 70 & 9,3 \\
\hline & Superior completo & 72 & 9,6 \\
\hline \multirow[t]{10}{*}{ Grau de instrução da mãe } & Não alfabetizado & 11 & 1,5 \\
\hline & Alfabetizado & 38 & 5,0 \\
\hline & Primário incompleto & 14 & 1,9 \\
\hline & Primário completo & 16 & 2,1 \\
\hline & Ginasial incompleto & 37 & 4,9 \\
\hline & Ginasial completo & 46 & 6,1 \\
\hline & $2^{\circ}$ Grau incompleto & 69 & 9,2 \\
\hline & 2 Grau completo & 345 & 45,8 \\
\hline & Superior incompleto & 70 & 9,3 \\
\hline & Superior completo & 107 & 14,2 \\
\hline \multirow[t]{6}{*}{ Habitação } & Própria quitada & 193 & 25,6 \\
\hline & Financiada & 239 & 31,7 \\
\hline & Cedida pelos pais & 88 & 11,7 \\
\hline & Cedida pelo trabalho & 7 & 0,9 \\
\hline & Alugada & 211 & 28,0 \\
\hline & Cedida & 15 & 2,0 \\
\hline \multirow[t]{3}{*}{ Ajuda do governo } & Não & 603 & 80,1 \\
\hline & Bolsa Família & 143 & 19,0 \\
\hline & Outra & 7 & 0,9 \\
\hline Com quem mora e a ocupação dos & Ambos empregados & 340 & 45,2 \\
\hline \multirow[t]{4}{*}{ Responsáveis } & Pai e mãe - Pai empregado & 133 & 17,7 \\
\hline & Pai e mãe - Mãe empregada & 42 & 5,6 \\
\hline & Ambos desempregados & 33 & 4,4 \\
\hline & Somente mãe - empregada & 101 & 13,4 \\
\hline
\end{tabular}


Tabela 1. Continuação...

\begin{tabular}{cccc}
\hline Variável & Categoria & Frequência & $\mathbf{\%}$ \\
\hline & Somente mãe - desempregada & 59 & 7,8 \\
& Somente pai - empregado & 44 & 5,8 \\
Com quem a criança fica & Somente pai - desempregado & 1 & 0,1 \\
& Mãe & 433 & 57,5 \\
Percepção do desempenho escolar & Pai & 59 & 7,8 \\
pelo responsável & Avós & 200 & 26,6 \\
& Vizinhos/Amigos & 25 & 3,3 \\
& Outros & 36 & 4,8 \\
& Excelente & 244 & 32,4 \\
& Muito bom & 254 & 33,7 \\
& Bom & 208 & 27,6 \\
\hline
\end{tabular}

Quanto à análise de saúde bucal, 44,5\% dos responsáveis classificaram a saúde bucal dos escolares como excelente ou muito boa e $35,3 \%$ como boa. 0 comportamento das crianças em relação à higiene bucal foi classificado pelos pais em 40,9\% como excelente ou muito boa, $36,5 \%$ como boa e $22,6 \%$ como regular ou ruim. Ainda, 2,9\% dos escolares apresentaram algum trauma dentário, segundo os responsáveis. Também, 58,4\% e 57,6\% dos escolares apresentavam má oclusão, de acordo com os índices de Foster e Hamilton e de Grabowisk, respectivamente. 0 CPOD médio da amostra foi de 1,33 com desvio padrão de 2,14, mínimo de zero e máximo de 13 (Tabela 2).

Tabela 2. Análise descritiva da amostra dos escolares da cidade de Araraquara, São Paulo, Brasil, quanto às variáveis relacionadas à saúde bucal $(n=753)$

\begin{tabular}{cccc}
\hline Variável & Categoria & Frequência & \% \\
\hline & Excelente & 119 & 15,8 \\
Percepção do responsável & Muito boa & 216 & 28,7 \\
sobre a saúde bucal da criança & Boa & 266 & 35,3 \\
& Regular & 124 & 16,5 \\
& Ruim & 28 & 3,7 \\
Percepção do responsável & Excelente & 120 & 15,9 \\
sobre a higiene bucal da & Muito bom & 188 & 25,0 \\
criança (comportamento) & Boa & 275 & 36,5 \\
& Regular & 146 & 19,4 \\
Trauma dentário & Ruim & 24 & 3,2 \\
Tratamento ortodôntico & Ausente & 731 & 97,1 \\
& Presente & 22 & 2,9 \\
& Ausente & 753 & 100,0 \\
Relação anteroposterior & Presente & 0 & 0,0 \\
& Classe I & 574 & 76,2 \\
& Classe II & 94 & 12,5 \\
& Classe III & 42 & 5,6 \\
& Assimétrica & 43 & 13,0 \\
& Normal & 444 & 11,2 \\
\hline
\end{tabular}


Tabela 2. Continuação...

\begin{tabular}{|c|c|c|c|}
\hline Variável & Categoria & Frequência & $\%$ \\
\hline \multirow{4}{*}{ Sobremordida } & Normal & 417 & 55,4 \\
\hline & Reduzida & 176 & 23,4 \\
\hline & Aberta & 59 & 7,8 \\
\hline & Profunda & 101 & 13,4 \\
\hline \multirow{2}{*}{ Mordida cruzada Posterior } & Ausência & 718 & 95,4 \\
\hline & Presença & 35 & 4,6 \\
\hline \multirow{3}{*}{$\begin{array}{l}\text { Má oclusão pelo Índice de } \\
\text { Foster }\end{array}$} & Ausência & 313 & 41,6 \\
\hline & Presença & 440 & 58,4 \\
\hline & Classe I & 574 & 76,2 \\
\hline \multirow{4}{*}{ Chave de canino } & Classe II & 93 & 12,4 \\
\hline & Classe III & 43 & 5,7 \\
\hline & Assimétrica & 43 & 5,7 \\
\hline & Normal & 530 & 70,4 \\
\hline \multirow[t]{3}{*}{ Sobressaliência } & Aumentada & 132 & 17,5 \\
\hline & Diminuída & 91 & 12,1 \\
\hline & Normal & 423 & 56,2 \\
\hline \multirow[t]{3}{*}{ Sobremordida } & Aumentada & 109 & 14,5 \\
\hline & Diminuída & 221 & 29,4 \\
\hline & Normal & 719 & 95,5 \\
\hline \multirow{3}{*}{$\begin{array}{l}\text { Trespasse transversal } \\
\text { posterior }\end{array}$} & Mordida cruzada unilateral & 26 & 3,4 \\
\hline & Mordida cruzada bilateral & 6 & 0,8 \\
\hline & Tesoura & 2 & 0,3 \\
\hline Má oclusão pelo Índice de & Ausência & 319 & 42,4 \\
\hline Grabowisk & Presença & 434 & 57,6 \\
\hline \multirow{3}{*}{ Má oclusão por um dos índices } & Ausência & 311 & 41,3 \\
\hline & Presença & 442 & 58,7 \\
\hline & & $\begin{array}{l}\text { Média (desvio } \\
\text { padrão) }\end{array}$ & $\begin{array}{l}\text { Mediana (mínimo e } \\
\text { máximo) }\end{array}$ \\
\hline CPOD & & $1,33(2,14)$ & $0,00(0,00-13,00)$ \\
\hline Índice de Foster & & $6,01(2,11)$ & $6,00(4,00-13,00)$ \\
\hline Índice de Grabowski & & $5,58(1,97)$ & $5,00(1,00-13,00)$ \\
\hline
\end{tabular}

Foi observado que, quanto à presença de hábitos orais deletérios, segundo os responsáveis, $12,0 \%$ das crianças apresentavam algum tipo de hábito oral deletério, sendo que 6,6\% apresentavam bruxismo, 1,5\% sucção de chupeta, 1,1\% mamadeira, 1,2\% tinha o hábito de sucção de dedo e apenas $0,3 \%$ apresentou hábito de sucção de língua, onicofagia e hábito de morder objetos (Tabela 3).

Tabela 3. Análise descritiva da amostra quanto à presença de hábitos orais deletérios na percepção dos responsáveis (Araraquara, São Paulo, Brasil) $(n=753)$

\begin{tabular}{cccc}
\hline Variável & Categoria & Frequência & Porcentagem \\
\hline \multirow{2}{*}{ Algum hábito } & Ausente & 663 & $88,0 \%$ \\
& Presente & 90 & $12,0 \%$ \\
\multirow{2}{*}{ Bruxismo } & Ausente & 703 & $93,4 \%$ \\
& Presente & 50 & $6,6 \%$ \\
Sucção de Chupeta & Ausente & 742 & $98,5 \%$ \\
\hline
\end{tabular}


Tabela 3. Continuação...

\begin{tabular}{cccc}
\hline Variável & Categoria & Frequência & Porcentagem \\
\hline \multirow{3}{*}{ Sucção de dedo } & Presente & 11 & $1,5 \%$ \\
& Ausente & 744 & $98,8 \%$ \\
& Presente & 9 & $1,2 \%$ \\
Sucção de língua & Ausente & 751 & $99,7 \%$ \\
& Presente & 2 & $0,3 \%$ \\
\multirow{2}{*}{ Uso de mamadeira } & Ausente & 745 & $98,9 \%$ \\
& Presente & 8 & $1,1 \%$ \\
Onicofagia & Ausente & 751 & $99,7 \%$ \\
& Presente & 2 & $0,3 \%$ \\
Morder objetos & Ausente & 751 & $99,7 \%$ \\
& Presente & 2 & $0,3 \%$ \\
\hline
\end{tabular}

Quanto às variáveis que foram analisadas individualmente em relação ao desempenho escolar percebido pelos responsáveis (Tabela 4), as mesmas apresentaram associação significativa entre sexo, renda da família, número de pessoas na família, grau de instrução do pai e da mãe, recebimento de ajuda financeira do governo, com quem a criança mora, ocupação dos responsáveis, com quem a criança fica quando não está na escola, percepção dos responsáveis sobre a saúde bucal da criança e percepção dos responsáveis sobre o comportamento da criança quanto à higiene bucal e experiência de cárie $(p<0,05)$. $\mathrm{Na}$ análise múltipla, ou seja, com ajuste entre as variáveis, observou-se que crianças de famílias com menores rendas (OR=1,76; IC95\%: 1,21-2,57; $\mathrm{p}=0,0032$ ), com pior saúde bucal, segundo a percepção dos responsáveis ( $\mathrm{OR}=1,93$; IC95\%: 1,27-2,93; $\mathrm{p}=0$,0022) e pior comportamento em relação à higiene oral (OR=6,60; IC95\%: 4.12-10,58; $\mathrm{p}<0,0001)$ têm mais chance de ter pior desempenho escolar, segundo a percepção dos responsáveis $(\mathrm{p} \leq 0,05)$ (Tabela 4).

Tabela 4. Análises (brutas e ajustadas) das associações com o desempenho escolar nos últimos 12 meses, segundo a percepção do responsável (Araraquara, São Paulo, Brasil) $(n=753)$

\begin{tabular}{|c|c|c|c|c|c|c|c|c|}
\hline \multirow{3}{*}{ Variáveis } & \multirow{3}{*}{ Categoria } & \multirow{3}{*}{ N (\%) } & \multicolumn{2}{|c|}{ Desempenho escolar } & \multirow{3}{*}{$\begin{array}{l}\text { OR bruto } \\
\text { (IC95\%) }\end{array}$} & \multirow{3}{*}{ p-valor } & \multirow{3}{*}{$\begin{array}{c}\text { OR } \\
\text { ajustado } \\
\text { (IC95\%) }\end{array}$} & \multirow{3}{*}{ p-valor } \\
\hline & & & Excelente/Muito bom & "Bom/Regular/Ruim & & & & \\
\hline & & & n (\%) & n (\%) & & & & \\
\hline \multicolumn{9}{|c|}{ Socio demográficas } \\
\hline \multirow{2}{*}{ Sexo } & Feminino & $\begin{array}{c}391 \\
(51,9 \%)\end{array}$ & $272(69,6 \%)$ & $119(30,4 \%)$ & Ref & & & \\
\hline & Masculino & $\begin{array}{c}362 \\
(48,1 \%)\end{array}$ & $226(62,4 \%)$ & $136(37,6 \%)$ & $\begin{array}{c}1,38 \\
(1,02-1,86)\end{array}$ & 0,0390 & & \\
\hline \multirow{2}{*}{ Renda } & Até 3 SM & $\begin{array}{c}471 \\
(62,6 \%)\end{array}$ & $278(59,0 \%)$ & $193(41,0 \%)$ & $\begin{array}{c}2,46 \\
(1,76-3,45)\end{array}$ & $<0,0001$ & $\begin{array}{c}1,76 \\
(1,21-2,57)\end{array}$ & 0,0032 \\
\hline & $\begin{array}{c}\text { Acima de } 3 \\
\text { SM }\end{array}$ & $\begin{array}{c}282 \\
(37,4 \%)\end{array}$ & $220(78,0 \%)$ & $62(22,0 \%)$ & Ref & & Ref & \\
\hline \multirow[t]{2}{*}{$\begin{array}{l}\text { Número de } \\
\text { pessoas }\end{array}$} & Até 4 & $\begin{array}{c}552 \\
(73,3 \%)\end{array}$ & $378(68,5 \%)$ & $174(31,5 \%)$ & Ref & & & \\
\hline & Acima de 4 & $\begin{array}{c}201 \\
(26,7 \%)\end{array}$ & $120(59,7 \%)$ & $81(40,3 \%)$ & $\begin{array}{c}1,47 \\
(1,05-2,05)\end{array}$ & 0,0248 & & \\
\hline \multirow[t]{2}{*}{$\begin{array}{c}\text { Grau de } \\
\text { instrução do pai }\end{array}$} & $\begin{array}{c}\text { Fundamen } \\
\text { tal }\end{array}$ & $\begin{array}{c}192 \\
(25,5 \%)\end{array}$ & $104(54,2 \%)$ & $88(45,8 \%)$ & $\begin{array}{c}2,00 \\
(1,43-2,80)\end{array}$ & $<0,0001$ & & \\
\hline & Acima & $\begin{array}{c}561 \\
(74,5 \%)\end{array}$ & $394(70,2 \%)$ & $167(29,8 \%)$ & Ref & & & \\
\hline
\end{tabular}


Tabela 4. Continuação...

\begin{tabular}{|c|c|c|c|c|c|c|c|c|}
\hline \multirow{3}{*}{ Variáveis } & \multirow{3}{*}{ Categoria } & \multirow{3}{*}{ N (\%) } & \multicolumn{2}{|c|}{ Desempenho escolar } & \multirow{3}{*}{$\begin{array}{l}\text { OR bruto } \\
\text { (IC95\%) }\end{array}$} & \multirow{3}{*}{ p-valor } & \multirow{3}{*}{$\begin{array}{c}\text { OR } \\
\text { ajustado } \\
\text { (IC95\%) }\end{array}$} & \multirow{3}{*}{ p-valor } \\
\hline & & & Excelente/Muito bom & ${ }^{*}$ Bom/Regular/Ruim & & & & \\
\hline & & & n (\%) & n (\%) & & & & \\
\hline \multirow[t]{3}{*}{$\begin{array}{c}\text { Grau de } \\
\text { instrução da } \\
\text { mãe }\end{array}$} & $\begin{array}{c}\text { Fundamen } \\
\text { tal }\end{array}$ & $\begin{array}{c}162 \\
(21,5 \%)\end{array}$ & $88(54,3 \%)$ & $74(45,7 \%)$ & $\begin{array}{c}1,90 \\
(1,34-2,72)\end{array}$ & 0,0004 & & \\
\hline & Acima & $\begin{array}{c}591 \\
(78,5 \%)\end{array}$ & $410(69,4 \%)$ & $181(30,6 \%)$ & Ref & & & \\
\hline & $\begin{array}{l}\text { Própria } \\
\text { quitada }\end{array}$ & $\begin{array}{c}193 \\
(25,6 \%)\end{array}$ & $129(66,8 \%)$ & $64(33,2 \%)$ & Ref & & & \\
\hline Habitação & Outros & $\begin{array}{c}560 \\
(74,4 \%)\end{array}$ & $369(65,9 \%)$ & $191(34,1 \%)$ & $\begin{array}{c}1,04 \\
(0,74-1,48)\end{array}$ & 0,8113 & & \\
\hline \multirow[t]{2}{*}{$\begin{array}{l}\text { Ajuda do } \\
\text { governo }\end{array}$} & Não & $\begin{array}{c}603 \\
(80,1 \%)\end{array}$ & $419(69,5 \%)$ & $184(30,5 \%)$ & Ref & & & \\
\hline & Sim & $\begin{array}{c}150 \\
(19,9 \%)\end{array}$ & $79(52,7 \%)$ & $71(47,3 \%)$ & $\begin{array}{c}2,05 \\
(1,42-2,95)\end{array}$ & 0,0001 & & \\
\hline \multirow[t]{2}{*}{$\begin{array}{c}\text { Com quem } \\
\text { mora/ } \\
\text { Ocupação }\end{array}$} & $\begin{array}{c}\text { Ambos } \\
\text { empregad } \\
\text { os }\end{array}$ & $\begin{array}{c}340 \\
(45,2 \%)\end{array}$ & $247(72,6 \%)$ & $93(27,4 \%)$ & Ref & & & \\
\hline & Outros & $\begin{array}{c}413 \\
(54,8 \%)\end{array}$ & $251(60,8 \%)$ & $162(39,2 \%)$ & $\begin{array}{c}1,71 \\
(1,26-2,34)\end{array}$ & 0,0006 & & \\
\hline \multirow[t]{2}{*}{$\begin{array}{l}\text { Com que a } \\
\text { criança fica }\end{array}$} & Mãe/Pai & $\begin{array}{c}492 \\
(65,3 \%)\end{array}$ & $338(68,7 \%)$ & $154(31,3 \%)$ & Ref & & & \\
\hline & Outros & $\begin{array}{c}261 \\
(34,7 \%)\end{array}$ & $160(61,3 \%)$ & $101(38,7 \%)$ & $\begin{array}{c}1,38 \\
(1,01-1,90)\end{array}$ & 0,0416 & & \\
\hline \multicolumn{9}{|l|}{ Saúde bucal } \\
\hline \multirow[t]{2}{*}{$\begin{array}{l}\text { Percepção do } \\
\text { responsável } \\
\text { sobre saúde } \\
\text { bucal }\end{array}$} & $\begin{array}{l}\text { Excelente/ } \\
\text { Muito boa }\end{array}$ & $\begin{array}{c}335 \\
(44,5 \%)\end{array}$ & $283(84,5 \%)$ & $52(15,5 \%)$ & Ref & & Ref & \\
\hline & $\begin{array}{l}\text { Boa/Regul } \\
\text { ar/Ruim }\end{array}$ & $\begin{array}{c}418 \\
(55,5 \%)\end{array}$ & $215(51,4 \%)$ & $203(48,6 \%)$ & $\begin{array}{c}5,14 \\
(3,61-7,31)\end{array}$ & $<0,0001$ & $\begin{array}{c}1,93 \\
(1,27-2,93)\end{array}$ & 0,0022 \\
\hline \multirow[t]{3}{*}{$\begin{array}{l}\text { Percepção do } \\
\text { responsável sobre } \\
\text { a higiene bucal }\end{array}$} & $\begin{array}{l}\text { Excelente/ } \\
\text { Muito boa }\end{array}$ & $\begin{array}{c}308 \\
(40,9 \%)\end{array}$ & $279(90,6 \%)$ & $29(9,4 \%)$ & Ref & & Ref & \\
\hline & $\begin{array}{l}\text { Boa/Regul } \\
\text { ar/Ruim }\end{array}$ & $\begin{array}{c}445 \\
(59,1 \%)\end{array}$ & $219(49,2 \%)$ & $226(50,8 \%)$ & $\begin{array}{c}9,93 \\
(6,49- \\
15,19)\end{array}$ & $<0,0001$ & $\begin{array}{r}6,60 \\
(4.12- \\
10,58)\end{array}$ & $<0,0001$ \\
\hline & Ausente & $\begin{array}{c}731 \\
(97,1 \%)\end{array}$ & $481(65,8 \%)$ & $250(34,2 \%)$ & Ref & & & \\
\hline Trauma dentário & Presente & $22(2,9 \%)$ & $17(77,3 \%)$ & $5(22,7 \%)$ & $\begin{array}{c}0,57 \\
(0,21-1,55)\end{array}$ & 0,2920 & & \\
\hline \multirow{2}{*}{$\begin{array}{l}\text { Experiência de } \\
\text { cárie }\end{array}$} & Ausência & $\begin{array}{c}447 \\
(59,4 \%)\end{array}$ & $309(69,1 \%)$ & $138(30,9 \%)$ & Ref & & & \\
\hline & Presença & $\begin{array}{c}306 \\
(40,6 \%)\end{array}$ & $189(61,8 \%)$ & $117(38,2 \%)$ & $\begin{array}{c}1,39 \\
(1,02-1,88)\end{array}$ & 0,0363 & & \\
\hline \multirow[t]{2}{*}{$\begin{array}{l}\text { Má oclusão pelo } \\
\text { Índice de Foster }\end{array}$} & Ausência & $\begin{array}{c}313 \\
(41,6 \%)\end{array}$ & $198(63,3 \%)$ & $115(36,7 \%)$ & Ref & & & \\
\hline & Presença & $\begin{array}{c}440 \\
(58,4 \%)\end{array}$ & $300(68,2 \%)$ & $140(31,8 \%)$ & $\begin{array}{c}0,80 \\
(0,59-1,09)\end{array}$ & 0,1598 & & \\
\hline \multirow{2}{*}{ Índice de Foster } & $\leq 6^{\&}$ & $\begin{array}{c}423 \\
(56,2 \%)\end{array}$ & $276(65,2 \%)$ & $147(34,8 \%)$ & Ref & & & \\
\hline & $>6$ & $\begin{array}{c}330 \\
(43,8 \%)\end{array}$ & $222(67,3 \%)$ & $108(32,7 \%)$ & $\begin{array}{c}0,91 \\
(0,67-1,24)\end{array}$ & 0,5603 & & \\
\hline
\end{tabular}


Tabela 4. Continuação...

\begin{tabular}{|c|c|c|c|c|c|c|c|c|}
\hline \multirow{3}{*}{ Variáveis } & \multirow{3}{*}{ Categoria } & \multirow{3}{*}{ N (\%) } & \multicolumn{2}{|c|}{ Desempenho escolar } & \multirow{3}{*}{$\begin{array}{l}\text { OR bruto } \\
\text { (IC95\%) }\end{array}$} & \multirow{3}{*}{ p-valor } & \multirow{3}{*}{$\begin{array}{c}\text { OR } \\
\text { ajustado } \\
\text { (IC95\%) }\end{array}$} & \multirow{3}{*}{ p-valor } \\
\hline & & & Excelente/Muito bom & *Bom/Regular/Ruim & & & & \\
\hline & & & n (\%) & n (\%) & & & & \\
\hline \multirow{4}{*}{$\begin{array}{c}\text { Má oclusão pelo } \\
\text { Grabowski }\end{array}$} & 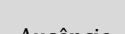 & 319 & & & & & & \\
\hline & Ausencia & $(42,4 \%)$ & $203(63,6 \%)$ & $116(36,4 \%)$ & Ret & & & \\
\hline & Precenco & 434 & $205(6800)$ & 120 & 0,82 & 02144 & & \\
\hline & rieseliça & $(57,6 \%)$ & $230(00,0 \%)$ & $139(32,0 \%)$ & $(0,61-1,12)$ & 0,2144 & & \\
\hline \multirow{4}{*}{$\begin{array}{l}\text { Índice de } \\
\text { Grabowski }\end{array}$} & & 448 & & & & & & \\
\hline & 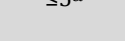 & $(59,5 \%)$ & $230(04,1 \%)$ & $150(35,3 \%)$ & Ker & & & \\
\hline & $>5$ & 305 & ) & $07(21000)$ & 0,86 & 02242 & & \\
\hline & -3 & $(40,5 \%)$ & $200(00,2 \%)$ & प्रा $(31,8 \%)$ & $(0,63-1,17)$ & 0,3242 & & \\
\hline \multirow{4}{*}{$\begin{array}{l}\text { Má oclusão por } \\
\text { um dos índices }\end{array}$} & Aucôncin & 311 & $100(62700$ & $112(26201$ & $\mathrm{Dof}$ & & & \\
\hline & Нusciteda & $(41,3 \%)$ & 1000,100 & $110(30,0)$ & KC1 & & & \\
\hline & Presenca & 442 & $300(670 \%)$ & $142(3210 \%$ & 0,83 & 02299 & & \\
\hline & 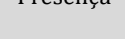 & $(58,7 \%)$ & (0) & $172(32,170)$ & $(0,61-1,13)$ & 0, & & \\
\hline \multicolumn{9}{|l|}{ Hábitos orais } \\
\hline \multirow{6}{*}{$\begin{array}{l}\text { Hábitos orais } \\
\text { deletérios }\end{array}$} & Ausência & 663 & $430(6620)$ & $22482380 \%$ & Ref & & & \\
\hline & Husticta & $(88,0 \%)$ & $403(00,2 \%)$ & $224(30,0 \%)$ & Ker & & & \\
\hline & Presenca & $90(120 \%)$ & $59(656 \%)$ & $31(34,4 \%)$ & 1,03 & 09011 & & \\
\hline & & & & & $(0,65-1,64)$ & & & \\
\hline & Aucente & 703 & & & & & & \\
\hline & RUSEIIL & $(93,4 \%)$ & $400(00,5 \%)$ & $23(53,1 \%)$ & NeI & & & \\
\hline \multirow{2}{*}{ Bruxismo } & & & & & 1,11 & & & \\
\hline & Presente & $50(0,6 \%)$ & $32(04,0 \%)$ & $18(30,0 \%)$ & $(0,61-2,01)$ & $0, / 413$ & & \\
\hline
\end{tabular}

*Categoria de referência para a variável de desfecho. Ref: Categoria de referência para as variáveis independentes; OR: Odds ratio; IC: Intervalo de confiança; \&mediana da amostra; SM= salário-mínimo. Ref: Categoria de referência para as variáveis exploratórias. AIC $($ modelo vazio $)=966,04 ;$ AIC $($ modelo final $)=794,14) ;-2 \log \mathrm{L}($ modelo vazio $)=964,04 ;-2 \log \mathrm{L}($ modelo final $)=786,14$.

Foi também construído um modelo, sem considerar as variáveis exploratórias de percepção dos pais (Tabela 5). A partir desse modelo, observa-se que crianças do sexo masculino (OR=1,48; IC95\%: 1,08-2,03; $p=0,0137)$, de famílias com menores rendas $(\mathrm{OR}=2,22$; IC05\%: 1,56-3,16; $p<0,0001)$, que recebem ajuda financeira do governo (OR=1,70; IC95\%: $1,16-2,50 ; p=0,0066$ ) e que ficam com outras pessoas que não o pai e/ou mãe quando não estão na escola (OR: 1,45; IC95\%1,04-2,00; $p=0,0263$ ) apresentam mais chance de ter pior desempenho escolar, segundo a percepção dos responsáveis, $p<0,05$. Não houve evidências de associação entre as variáveis de saúde bucal e de hábitos orais deletérios com o desempenho escolar percebido pelos responsáveis ( $\mathrm{p}>0,05)$.

Tabela 5. Análises (brutas e ajustadas) das associações com o desempenho escolar nos últimos 12 meses, desconsiderando a percepção do responsável ( $n=753$ ) (Araraquara, São Paulo, Brasil)

\begin{tabular}{|c|c|c|c|c|c|c|c|c|}
\hline \multirow[b]{2}{*}{ Variáveis } & \multirow[b]{2}{*}{ Categoria } & \multirow[b]{2}{*}{ N (\%) } & \multicolumn{2}{|c|}{ Desempenho escolar } & \multirow{2}{*}{$\begin{array}{l}\text { OR bruto } \\
\text { (IC95\%) }\end{array}$} & \multirow[b]{2}{*}{ p-valor } & \multirow{2}{*}{$\begin{array}{c}\text { OR ajustado } \\
\text { (IC95\%) }\end{array}$} & \multirow[b]{2}{*}{ p-valor } \\
\hline & & & $\begin{array}{c}\text { Excelente/ } \\
\text { Muito bom } \\
\mathbf{n}(\%)\end{array}$ & $\begin{array}{c}{ }^{*} \text { Bom/Reg } \\
\text { ular/Ruim } \\
\text { n (\%) } \\
\end{array}$ & & & & \\
\hline \multicolumn{9}{|c|}{ Sócio- demográficas } \\
\hline \multirow[t]{2}{*}{ Sexo } & Feminino & $391(51,9 \%)$ & $272(69,6 \%)$ & $\begin{array}{c}119 \\
(30,4 \%)\end{array}$ & Ref & & Ref & \\
\hline & Masculino & $362(48,1 \%)$ & $226(62,4 \%)$ & $\begin{array}{c}136 \\
(37,6 \%)\end{array}$ & $\begin{array}{c}1,38 \\
(1,02-1,86)\end{array}$ & 0,0390 & $\begin{array}{c}1,48 \\
(1,08-2,03)\end{array}$ & 0,0137 \\
\hline
\end{tabular}


Tabela 5. Continuação...

\begin{tabular}{|c|c|c|c|c|c|c|c|c|}
\hline \multirow[b]{2}{*}{ Variáveis } & \multirow[b]{2}{*}{ Categoria } & \multirow[b]{2}{*}{ N (\%) } & \multicolumn{2}{|c|}{ Desempenho escolar } & \multirow{2}{*}{$\begin{array}{l}\text { OR bruto } \\
\text { (IC95\%) }\end{array}$} & \multirow[b]{2}{*}{ p-valor } & \multirow{2}{*}{$\begin{array}{l}\text { OR ajustado } \\
\text { (IC95\%) }\end{array}$} & \multirow[b]{2}{*}{ p-valor } \\
\hline & & & $\begin{array}{c}\begin{array}{c}\text { Excelente/ } \\
\text { Muito bom }\end{array} \\
\mathbf{n}(\%)\end{array}$ & $\begin{array}{c}{ }^{*} \text { Bom/Reg } \\
\text { ular/Ruim } \\
\text { n (\%) }\end{array}$ & & & & \\
\hline \multirow[t]{2}{*}{ Salário-mínimo } & Até 3 & $471(62,6 \%)$ & $278(59,0 \%)$ & $\begin{array}{c}193 \\
(41,0 \%)\end{array}$ & $\begin{array}{c}2,46 \\
(1,76-3,45)\end{array}$ & $<0,0001$ & $\begin{array}{c}2,22 \\
(1,56-3,16)\end{array}$ & $<0,0001$ \\
\hline & Acima de 3 & $282(37,4 \%)$ & $\begin{array}{c}220 \\
(78,0 \%)\end{array}$ & $62(22,0 \%)$ & Ref & & Ref & \\
\hline \multirow[t]{2}{*}{ Número de pessoas } & Até 4 & $552(73,3 \%)$ & $\begin{array}{c}378 \\
(68,5 \%)\end{array}$ & $174(31,5 \%)$ & Ref & & & \\
\hline & Acima de 4 & $201(26,7 \%)$ & $\begin{array}{c}120 \\
(59,7 \%)\end{array}$ & $81(40,3 \%)$ & $\begin{array}{c}1,47 \\
(1,05-2,05)\end{array}$ & 0,0248 & & \\
\hline Grau de instrução & Fundamental & $192(25,5 \%)$ & $\begin{array}{c}104 \\
(54,2 \%)\end{array}$ & $88(45,8 \%)$ & $\begin{array}{c}2,00 \\
(1,43-2,80)\end{array}$ & $<0,0001$ & & \\
\hline do pai & Acima & $561(74,5 \%)$ & $\begin{array}{c}394 \\
(70,2 \%)\end{array}$ & $167(29,8 \%)$ & Ref & & & \\
\hline \multirow[t]{2}{*}{$\begin{array}{c}\text { Grau de instrução da } \\
\text { mãe }\end{array}$} & Fundamental & $162(21,5 \%)$ & $\begin{array}{c}88 \\
(54,3 \%)\end{array}$ & $74(45,7 \%)$ & $\begin{array}{c}1,90 \\
(1,34-2,72)\end{array}$ & 0,0004 & & \\
\hline & Acima & $591(78,5 \%)$ & $\begin{array}{c}410 \\
(69,4 \%)\end{array}$ & $181(30,6 \%)$ & Ref & & & \\
\hline \multirow[t]{2}{*}{ Habitação } & Própria quitada & $193(25,6 \%)$ & $\begin{array}{c}129 \\
(66,8 \%)\end{array}$ & $64(33,2 \%)$ & Ref & & & \\
\hline & Outros & $560(74,4 \%)$ & $\begin{array}{c}369 \\
(65,9 \%)\end{array}$ & $191(34,1 \%)$ & $\begin{array}{c}1,04 \\
(0,74-1,48)\end{array}$ & 0,8113 & & \\
\hline \multirow[t]{2}{*}{ Ajuda do governo } & Não & $603(80,1 \%)$ & $\begin{array}{c}419 \\
(69,5 \%)\end{array}$ & $184(30,5 \%)$ & Ref & & Ref & \\
\hline & Sim & $150(19,9 \%)$ & $\begin{array}{c}79 \\
(52,7 \%)\end{array}$ & $71(47,3 \%)$ & $\begin{array}{c}2,05 \\
(1,42-2,95)\end{array}$ & 0,0001 & $\begin{array}{c}1,70 \\
(1,16-2,50)\end{array}$ & 0,0066 \\
\hline \multirow[t]{2}{*}{$\begin{array}{l}\text { Com que mora/ } \\
\text { ocupação }\end{array}$} & $\begin{array}{c}\text { Ambos } \\
\text { empregados }\end{array}$ & $340(45,2)$ & $\begin{array}{c}247 \\
(72,6 \%)\end{array}$ & $93(27,4 \%)$ & Ref & & & \\
\hline & Outros & $413(54,8 \%)$ & $\begin{array}{c}251 \\
(60,8 \%)\end{array}$ & $162(39,2 \%)$ & $\begin{array}{c}1,71 \\
(1,26-2,34)\end{array}$ & 0,0006 & & \\
\hline \multirow[t]{2}{*}{$\begin{array}{c}\text { Com quem a criança } \\
\text { fica }\end{array}$} & Mãe/Pai & $492(65,3 \%)$ & $\begin{array}{c}338 \\
(68,7 \%)\end{array}$ & $154(31,3 \%)$ & Ref & & Ref & \\
\hline & Outros & $261(34,7 \%)$ & $\begin{array}{c}160 \\
(61,3 \%)\end{array}$ & $101(38,7 \%)$ & $\begin{array}{c}1,38 \\
(1,01-1,90)\end{array}$ & 0,0416 & $\begin{array}{c}1,45 \\
(1,04-2,00)\end{array}$ & 0,0263 \\
\hline \multicolumn{9}{|l|}{ Saúde bucal } \\
\hline \multirow[t]{2}{*}{ Trauma dentário } & Ausente & $731(97,1 \%)$ & 481 & $250(34,2 \%)$ & Ref & & & \\
\hline & Presente & $22(2,9 \%)$ & $\begin{array}{c}17 \\
(77,3 \%)\end{array}$ & $5(22,7 \%)$ & $\begin{array}{c}0,57 \\
(0,21-1,55)\end{array}$ & 0,2920 & & \\
\hline \multirow[t]{2}{*}{ Experiência de cárie } & Ausência & $447(59,4 \%)$ & $\begin{array}{c}309 \\
(69,1 \%)\end{array}$ & $138(30,9 \%)$ & Ref & & & \\
\hline & Presença & $306(40,6 \%)$ & $\begin{array}{c}189 \\
(61,8 \%)\end{array}$ & $117(38,2 \%)$ & $\begin{array}{c}1,39 \\
(1,02-1,88)\end{array}$ & 0,0363 & & \\
\hline
\end{tabular}


Tabela 5. Continuação...

\begin{tabular}{|c|c|c|c|c|c|c|c|c|}
\hline \multirow{3}{*}{ Variáveis } & \multirow{3}{*}{ Categoria } & \multirow{3}{*}{ N (\%) } & \multicolumn{2}{|c|}{ Desempenho escolar } & \multirow{3}{*}{$\begin{array}{l}\text { OR bruto } \\
\text { (IC95\%) }\end{array}$} & \multirow{3}{*}{ p-valor } & \multirow{3}{*}{$\begin{array}{l}\text { OR ajustado } \\
\text { (IC95\%) }\end{array}$} & \multirow{3}{*}{ p-valor } \\
\hline & & & $\begin{array}{l}\text { Excelente/ } \\
\text { Muito bom } \\
\end{array}$ & $\begin{array}{l}{ }^{*} \text { Bom/Reg } \\
\text { ular/Ruim } \\
\end{array}$ & & & & \\
\hline & & & n (\%) & n (\%) & & & & \\
\hline \multirow{4}{*}{$\begin{array}{l}\text { Má oclusão pelo Índice } \\
\text { de Foster }\end{array}$} & Ausência & $313(41,6 \%)$ & 198 & 115(36,7\%) & Ref & & & \\
\hline & & & $(63,3 \%)$ & & & & & \\
\hline & Presença & $440(58,4 \%)$ & 300 & $140(31,8 \%)$ & 0,80 & 0,1598 & & \\
\hline & & & $(68,2 \%)$ & & $(0,59-1,09)$ & & & \\
\hline \multirow[t]{4}{*}{ Índice de Foster } & $\leq 6^{\&}$ & $423(56,2 \%)$ & 276 & $147(34,8 \%)$ & Ref & & & \\
\hline & & & $(65,2 \%)$ & & & & & \\
\hline & $>6$ & $330(43,8 \%)$ & 222 & $108(32,7 \%)$ & 0,91 & 0,5603 & & \\
\hline & & & $(67,3 \%)$ & & $(0,67-1,24)$ & & & \\
\hline \multirow{4}{*}{$\begin{array}{c}\text { Má oclusão pelo } \\
\text { Grabowski }\end{array}$} & Ausência & $319(42,4 \%)$ & 203 & $116(36,4 \%)$ & Ref & & & \\
\hline & & & $(63,6 \%)$ & & & & & \\
\hline & Presença & $434(57,6 \%)$ & 295 & $139(32,0 \%)$ & 0,82 & 0,2144 & & \\
\hline & & & $(68,0 \%)$ & & $(0,61-1,12)$ & & & \\
\hline \multirow[t]{4}{*}{ Índice de Grabowski } & $\leq 5 \&$ & $448(59,5 \%)$ & 290 & $158(35,3 \%)$ & Ref & & & \\
\hline & & & $(64,7 \%)$ & & & & & \\
\hline & $>5$ & $305(40,5 \%)$ & 208 & $97(31,8 \%)$ & 0,86 & 0,3242 & & \\
\hline & & & $(68,2 \%)$ & & $(0,63-1,17)$ & & & \\
\hline \multirow{4}{*}{$\begin{array}{c}\text { Má oclusão por um dos } \\
\text { índices }\end{array}$} & Ausência & $311(41,3 \%)$ & 198 & $113(36,3 \%)$ & Ref & & & \\
\hline & & & $(63,7 \%)$ & & & & & \\
\hline & Presença & $442(58,7 \%)$ & 300 & $142(32,1 \%)$ & 0,83 & 0,2299 & & \\
\hline & & & $(67,9 \%)$ & & $(0,61-1,13)$ & & & \\
\hline \multicolumn{9}{|l|}{ Hábitos orais } \\
\hline \multirow{4}{*}{$\begin{array}{c}\text { Hábitos orais } \\
\text { deletérios }\end{array}$} & Ausência & $663(88,0 \%)$ & 439 & $224(33,8 \%)$ & Ref & & & \\
\hline & & & $(66,2 \%)$ & & & & & \\
\hline & Presença & $90(12,0 \%)$ & 59 & $31(34,4 \%)$ & 1,03 & 0,9011 & & \\
\hline & & & $(65,6 \%)$ & & $(0,65-1,64)$ & & & \\
\hline \multirow[t]{4}{*}{ Bruxismo } & Ausente & $703(93,4 \%)$ & 466 & $237(33,7 \%)$ & Ref & & & \\
\hline & & & $(66,3 \%)$ & & & & & \\
\hline & Presente & $50(6,6 \%)$ & 32 & $18(36,0 \%)$ & 1,11 & 0,7413 & & \\
\hline & & & $(64,0 \%)$ & & $(0,61-2,01)$ & & & \\
\hline
\end{tabular}

*Categoria de referência para a variável de desfecho. Ref: Categoria de referência para as variáveis independentes; OR: Odds ratio; IC: Intervalo de confiança; \&mediana da amostra; SM = salário-mínimo. Ref: Categoria de referência para as variáveis exploratórias. AIC (modelo vazio) = 966,04; AIC (modelo final) = 927,89; -2 Log L (modelo vazio) = 964,04; -2 Log L (modelo final) $=917,89$. 


\section{DISCUSSÃO}

O presente estudo avaliou as relações entre variáveis de condições socioeconômicas, saúde bucal, hábitos e má oclusão, e desempenho escolar de crianças de cinco anos pertencentes à rede municipal de ensino da cidade de Araraquara, São Paulo, Brasil.

Carminatti et al. ${ }^{11}$ (2017) verificaram a relação da cárie dentária, da má oclusão e dos hábitos orais deletérios com a qualidade de vida dos pré-escolares e encontraram impacto negativo na mesma, interferindo na autoimagem e na interação social, conforme dados estatísticos e a própria percepção dos pais ${ }^{11}$. Porém, mesmo sabendo que a má oclusão atrapalha a vida das crianças, não foram observadas associações entre a má oclusão e os impactos relacionados à saúde bucal dessas crianças. Já no estudo de Kramer et al.12 (2013), demonstrou-se que a prevalência de qualquer impacto na qualidade de vida para as crianças que apresentavam cárie foi afetada três vezes mais do que para as crianças que não apresentavam essa doença e 1,5 vez mais para as crianças que tinham má oclusão. 0 predomínio de crianças com má oclusão nos nossos achados foi de $57,6 \%$ dos escolares, o que corrobora com outro estudo que encontrou alta taxa de alterações oclusais $(87,4 \%)$, com impacto direto na qualidade de vida e interferindo em várias fases da vida da criança ${ }^{13}$.

Outros achados observaram a relação do desempenho escolar de crianças que frequentavam atendimento odontológico público e associaram com hábitos bucais parafuncionais, concluindo que não houve associação dos hábitos deletérios com o desempenho escolar na amostra avaliada. Esses dados corroboram com os da nossa pesquisa, na qual somente $12 \%$ das crianças apresentavam algum tipo de hábito oral deletério, não apresentando nenhuma associação entre as variáveis desses hábitos orais com o desempenho escolar das crianças avaliadas. ${ }^{* * *}$ No que se refere aos hábitos e suas consequências na cavidade bucal, quanto maior o tempo de aleitamento materno menor a presença de algum hábito oral deletério desenvolvido pela criança ${ }^{4}$. Destacouse que o tempo de amamentação é o que influencia no uso de chupeta e mamadeira ${ }^{14}$. Isso vai ao encontro a uma afirmação de um estudo, em que o uso recorrente da mamadeira mostrou causar alterações na sucção e na mastigação, podendo resultar em más oclusões futuras. ${ }^{* * *} \mathrm{Em}$ contrapartida, evidenciaram que o tempo de duração dos hábitos poderia estar associado à presença de alterações oclusais e na fala ${ }^{3}$.

Dentre os hábitos deletérios mais comumente encontrados, temos a sucção de chupeta e/ou dedo, interposição labial, respiração bucal, onicofagia e bruxismo. Cada um pode causar uma alteração diferente na cavidade bucal, sendo de extrema importância que eles sejam removidos precocemente para que a criança possa crescer e se desenvolver de forma adequada, evitando problemas futuros que possam interferir na sua qualidade de vida e, consequentemente, no seu desempenho escolar. Foi demonstrado por Boeck et al. ${ }^{13}$, em uma amostra de crianças de três a seis anos, que o hábito deletério mais frequente era a sucção de chupeta. Este dado leva a direções opostas dos nossos achados, em que apenas $1,5 \%$ da amostra fazia o uso da chupeta, sendo o hábito deletério mais recorrente o bruxismo (6,6\%). Acredita-se que o bruxismo do sono seja mais comum na infância e pode estar associado a fatores funcionais, estruturais e/ou psicológicos, podendo causar muitos danos ao sistema estomatognático. Além disso, é um hábito deletério que está diretamente relacionado à má qualidade do sono ${ }^{15}$.

Deve-se considerar que um hábito deletério muito comum, que interfere na qualidade do sono e leva à falta de concentração no desempenho de atividades diárias, é a respiração bucal. A criança que apresenta essa alteração tem dificuldade de dormir e sempre está exausta e sem ânimo, levando a um baixo desempenho escolar, além de isolamento social, o que reflete de forma direta na qualidade de vida $^{15}$. Entretanto, em nosso trabalho, o padrão de alteração de respiração não foi elucidado. A

*** Fonseca LAM. Desempenho escolar em crianças de 6 a 10 anos e associação de hábitos bucais deletérios [dissertação]. Piracicaba: Faculdade de Odontologia de Piracicaba; 2018.

**** Lopes AR. Alterações bucais causadas pelo uso prolongado de chupeta e mamadeira em crianças [trabalho de conclusão de curso]. Araçuaí: Faculdade de Medicina da Universidade Federal de Minas Gerais; 2010. 
respiração oral não influenciou o desempenho escolar das crianças ${ }^{5}$. Silva et al. ${ }^{16}$ demonstraram em préescolares que hábitos relacionados com a qualidade do sono acabam refletindo na qualidade de vida das crianças, como também no desempenho das atividades diárias.

Associações entre o desempenho escolar e o índice socioeconômico baixo ${ }^{* * * *}$ concordaram com os nossos achados, que mostraram que crianças de famílias com menor renda têm 1,76 vez mais chances de ter pior desempenho escolar. Resultados semelhantes foram encontrados em uma revisão sobre a influência da saúde bucal, como cárie, gengivite, trauma e dor, no desempenho escolar, na qual concluiu-se que crianças que possuíam alguma doença dentária podiam estar em desvantagem quanto ao desenvolvimento, fornecendo evidências de que a influência da condição de saúde bucal e a percepção negativa dos pais sobre esta comprometem o desempenho escolar. ${ }^{* * * * *}$ Rezende et al. ${ }^{17}$, também, demonstraram que o mau desempenho escolar está associado à pior autopercepção de saúde e às características sociodemográficas, criando um impacto negativo na qualidade de vida destas crianças.

Quando se trata de saúde geral, um estudo de Karande, Venkataraman ${ }^{18}$ elucidou que o fato de crianças possuírem saúde geral ruim ou muito ruim interferiu de forma direta na qualidade de vida das mesmas e, consequentemente, prejudicou o desempenho escolar principalmente no sexo feminino. Entretanto, em nosso estudo, constatou-se que crianças do sexo masculino mostraram $1,48 \%$ vez mais chance de ter pior desempenho escolar em relação às crianças do sexo feminino, corroborando com os achados de Osti, Martinelli19, segundo os quais meninos apresentaram desempenho insatisfatório no processo de aprendizagem, em relação às meninas.

No geral, crianças de baixa renda tendem a ter um acesso mais complicado aos cuidados com a saúde geral e, também, com a cavidade bucal, estando, por isso, mais sujeitas a sofrerem os efeitos negativos que isso pode causar, como: exclusão social, baixa autoestima e baixo rendimento escolar, com consequência direta na qualidade de vida. Em um estudo, o desempenho escolar ruim foi associado a um autorrelato de saúde bucal ruim ${ }^{20}$. Já em outros achados, o impacto das condições bucais e fatores socioeconômicos no desempenho escolar tem correlação positiva entre lesões de cárie não tratadas, fator socioeconômico e dificuldade de prestar atenção na aula. Em suma, os escolares que possuíam maior desordem bucal eram os mesmos que apresentavam baixo desempenho escolar ${ }^{21}$. Os nossos achados mostraram que crianças que apresentavam um comportamento deficiente em relação à higiene oral tiveram $6,6 \%$ vezes mais chances de ter pior desempenho escolar. Já outros estudos avaliaram o impacto da saúde bucal nas atividades diárias e a presença de dor associada às lesões de cárie, e não encontraram associação entre condições clínicas e desempenho escolar. ${ }^{* * * * * *}$

É importante deixar claro que nosso estudo possui limitações, como o fato de ser um estudo transversal, no qual dependemos da resposta fiel dos entrevistados no questionário. Assim, para minimizar este viés, foram realizados testes de concordância intraexaminadores em $10 \%$ da amostra. É de suma importância que mais estudos epidemiológicos sejam realizados com o intuito de embasar ações para melhorar a qualidade de vida das crianças, seja no âmbito escolar e/ou familiar, para que elas possam ter acesso a serviços que beneficiem o seu desenvolvimento sob todos os aspectos.

\section{CONCLUSÃO}

Concluiu-se que possuem mais chances de ter pior desempenho escolar, levando em conta a percepção dos pais, crianças de famílias com menores rendas mensais e com pior saúde e higiene bucal.

\footnotetext{
***** Fonseca LAM. Desempenho escolar em crianças de 6 a 10 anos e associação de hábitos bucais deletérios [dissertação]. Piracicaba: Faculdade de Odontologia de Piracicaba; 2018.

****** Quadros LN. Condições de saúde bucal e sua relação com desempenho e absenteísmo escolar de crianças e adolescentes: uma revisão sistemática [dissertação]. Manaus: Universidade Federal do Amazonas; 2019.

******* Almeida RF. Avaliação das condições de saúde bucal e sua relação com o desempenho escolar de crianças do Paranoá-DF [dissertação]. Brasília: Universidade de Brasília; 2017.
} 
Além disso, também possuem mais chances de pior desempenho escolar crianças de famílias que recebem auxílio financeiro e que ficam com outras pessoas que não os pais quando não estão na escola.

\section{AGRADECIMENTOS}

Agradecimento especial à Prefeitura Municipal de Araraquara, representada pelas Secretárias de Saúde e de Educação, que permitiu a coleta de dados nos CERs para que este trabalho pudesse ser realizado.

\section{REFERÊNCIAS}

1. Sigaud CHS, Santos BR, Costa P, Toriyama ATM. Promoting oral care in the preschool child: effects of a playful learning intervention. Rev Bras Enferm. 2017 May-Jun;70(3):519-25. http://dx.doi.org/10.1590/0034-7167-2016-0237. PMid:28562799.

2. Oliva MIG, Cunha IP, Silva AN, Mialhe FL, Cortellazzi KL, Meneghim MC, et al. Senso de coerência e fatores associados ao desempenho escolar de adolescentes. Cien Saude Colet. 2019 Ago;24(8):3057-66. http://dx.doi.org/10.1590/1413-81232018248.22642017. PMid:31389552.

3. Pereira TS, Oliveira F, Cardoso MCAF. Associação entre hábitos orais deletérios e as estruturas e funções do sistema estomatognático: percepção dos responsáveis. CoDAS. 2017;29(3):e20150301. http://dx.doi.org/10.1590/2317-1782/20172015301. PMid:28538822.

4. Fernandes DMZ, Lima MCMP. A visão dos pais e professores sobre a ocorrência de hábitos orais deletérios em um grupo de pré-escolares. Rev CEFAC. 2019;21(2):1-10.

5. Abreu ACB, Moreles DA, Ballo MBJF. A respiração oral influencia o rendimento escolar? Rev CEFAC. 2003;5:69-73.

6. Landis JR, Koch GG. The measurement of observer agreement for categorical data. Biometrics. 1977 Mar;33(1):159-74. http://dx.doi.org/10.2307/2529310. PMid:843571.

7. Assaf AV, Zanin L, Meneghim MC, Pereira AC, Ambrosano GMB. Comparação entre medidas de reprodutibilidade para a calibração em levantamentos epidemiológicos da cárie dentária. Cad Saude Publica. 2006 Set;22(9):1901-7. http://dx.doi.org/10.1590/S0102-311X2006000900021. PMid:16917587.

8. Foster TD, Hamilton MC. Occlusion in the primary dentition. Study of children at 2 and one-half to 3 years of age. Br Dent J. 1969 Jan;126(2):76-9. PMid:5253447.

9. Dimberg L, Lennartsson B, Arnrup K, Bondemark L. Prevalence and change of malocclusions from primary to early permanent dentition: a longitudinal study. Angle Orthod. 2015 Sep;85(5):728-34. http://dx.doi.org/10.2319/080414-542.1. PMid:25867255.

10. Meneghim MC, Kozlowski FC, Pereira AC, Ambrosano GMB, Meneghim ZMAP. Classificação socioeconômica e sua discussão em relação à prevalência de cárie e fluorose dentária. Cien Saude Colet. 2007 Abr;12(2):523-9. http://dx.doi.org/10.1590/S1413-81232007000200028. PMid:17680106.

11. Carminatti M, Lavra-Pinto B, Franzon R, Rodrigues JA, Araújo FB, Gomes E. Impacto da cárie dentária, maloclusão e hábitos orais na qualidade de vida relacionada à saúde oral em crianças pré-escolares. Audiol Commun Res. 2017;22:1-8. http://dx.doi.org/10.1590/2317-6431-2016-1801.

12. Kramer PF, Feldens CA, Ferreira SH, Bervian J, Rodrigues PH, Peres MA. Exploring the impact of oral diseases and disorders on quality of life of preschool children. Community Dent Oral Epidemiol. 2013 Aug;41(4):327-35. http://dx.doi.org/10.1111/cdoe.12035. PMid:23330729. 
13. Boeck EM, Pizzol KEDC, Barbosa EGP, Pires NCA, Lunardi N. Prevalência de má oclusão em crianças de 3 a 6 anos portadoras de hábito de sucção de dedo e/ou chupeta. Rev Odontol UNESP. 2013 MarApr;42(2):110-6. http://dx.doi.org/10.1590/S1807-25772013000200008.

14. Capucho BL, Forechi L, Lima RCD, Massoroni L, Primo CC. Fatores que interferem na amamentação exclusiva. Rev Bras Pesq Saúde. 2017 Jan-Mar;19(1):108-13.

15. Simões-Zenari MS, Bitar ML. Fatores associados ao bruxismo em crianças de 4 a 6 anos. Pró-Fono R Atual Cient. 2010 Dez;22(4):465-72. http://dx.doi.org/10.1590/S0104-56872010000400018.

16. Silva EMB, Simões PAD, Macedo MCSA, Duarte JC, Silva DM. Percepção parental sobre hábitos e qualidade do sono das crianças em idade pré-escolar. Referência. 2018;4(17):63-71.

17. Rezende BA, Lemos SMA, Medeiros AM. Qualidade de vida e autopercepção de saúde de crianças com mau desempenho escolar. Rev Paul Pediatr. 2017 Oct-Dec;35(4):415-21. http://dx.doi.org/10.1590/1984-0462/;2017;35;4;00009. PMid:29185623.

18. Karande S, Venkataraman R. Self-perceived health-related quality of life of Indian children with specific learning disability. J Postgrad Med. 2012 Oct-Dec;58(4):246-54. http://dx.doi.org/10.4103/0022-3859.105442. PMid:23298918.

19. Osti A, Martinelli SC. Desempenho escolar: análise comparativa em função do sexo e percepção dos estudantes. Educ Pesqui. 2014 Mar;40(1):49-59. http://dx.doi.org/10.1590/S151797022013005000021.

20. David J, Åstrom AN, Wang NJ. Prevalence and correlates of self-reported state of teeth among schoolchildren in Kerala, India. BMC Oral Health. 2006;6(1):10. http://dx.doi.org/10.1186/14726831-6-10. PMid:16817952.

21. Paula JS, Lisboa CM, Meneghim MC, Pereira AC, Ambrosano GMB, Mialhe FL. School performance and oral health conditions: analysis of the impact mediated by socio-economic factors. Int J Paediatr Dent. 2016 Jan;26(1):52-9. http://dx.doi.org/10.1111/ipd.12158. PMid:25752583.

\section{CONFLITOS DE INTERESSE}

Os autores declaram não haver conflitos de interesse.

\section{*AUTOR PARA CORRESPONDÊNCIA}

Eloisa Marcantonio, UNIARA - Universidade de Araraquara, Faculdade de Odontologia, Departamento de Ortodontia, Avenida Maria Antônia Camargo de Oliveira, 170, CEP 14807-120 Araraquara - SP, Brasil, e-mail: eloisamarcantonio@gmail.com

Recebido: Novembro 3, 2021

Aprovado: Novembro 4, 2021 